\title{
COVID-19 and lung ultrasound: reasons why pediatricians can support adult COVID-19 units during critical epidemiologic periods
}

\author{
Cristina De Rose ${ }^{1}$, Luca Pierantoni ${ }^{2}$, and Danilo Buonsenso ${ }^{3}$ \\ ${ }^{1}$ Universita Cattolica del Sacro Cuore Sede di Roma \\ ${ }^{2}$ Pediatric Emergency Unit, S. Orsola University Hospital, Bologna, Italy \\ ${ }^{3}$ University Hospital Agostino Gemelli
}

March 20, 2021

\begin{abstract}
The massive raise of COVID-19 cases all over the world is leading to unprecedented pressures on healthcare services. Growing evidence is highlighting that COVID-19 is a systemic condition that requires doctors with multiple expertise. Paediatricians are trained in these skills. Considering the issue of staff shortage that is facing every country in the world, and the complexity of COVID-19, paediatricians may represent an important source of ready and skilled specialists that can quickly translate the paediatric practice in the COVID-19 care. We report our experience of paediatricians engaged in the fight against COVID-19 within adult COVID-19 wards. In particular, we focus on the importance of a skill of some paediatricians in their clinical practice, namely the use of lung ultrasound which has also become fundamental in the management of adult patients with COVID-19, especially for some categories such as pregnant women.
\end{abstract}

COVID-19 and lung ultrasound: reasons why pediatricians can support adult COVID-19 units during critical epidemiologic periods

Cristina De Rose ${ }^{1}$, MD; Luca Pierantoni², MD; Danilo Buonsenso ${ }^{1,3,4^{*}}$, MD

${ }^{1}$ Department of Woman and Child Health and Public Health, Fondazione Policlinico Universitario A. Gemelli IRCCS, Rome, Italy

${ }^{2}$ Pediatric Emergency Unit, S. Orsola University Hospital, Bologna, Italy

${ }^{3}$ Global Health Research Center, Università Cattolica del Sacro Cuore, Roma, Italia

${ }^{4}$ Istituto di Microbiologia, Università Cattolica del Sacro Cuore, Roma, Italia

*Corresponding Author

\section{Danilo Buonsenso}

Department of Woman and Child Health and Public Health, University Hospital Agostino Gemelli, Via Largo Agostino Gemelli 8, Rome, Italy

tel.: +393319819715 - +390630154475/4390

fax: +39063383211

email:danilobuonsenso@gmail.com

Twitter: @surf4children 


\title{
Running Head: Pediatricians and their support to adult COVID-19 unites
}

Conflicts of interest: None of the participants has any conflict of interest to declare.

Funding: Nothing to declare.

Key words: COVID-19, shortage of health workers, pediatricians, to support adult COVID-19 unites, lung ultrasound, LUS

\begin{abstract}
The massive raise of COVID-19 cases all over the world is leading to unprecedented pressures on healthcare services.

Growing evidence is highlighting that COVID-19 is a systemic condition that requires doctors with multiple expertise. Paediatricians are trained in these skills. Considering the issue of staff shortage that is facing every country in the world, and the complexity of COVID-19, paediatricians may represent an important source of ready and skilled specialists that can quickly translate the paediatric practice in the COVID-19 care.
\end{abstract}

We report our experience of paediatricians engaged in the fight against COVID-19 within adult COVID-19 wards. In particular, we focus on the importance of a skill of some paediatricians in their clinical practice, namely the use of lung ultrasound which has also become fundamental in the management of adult patients with COVID-19, especially for some categories such as pregnant women.

Since the beginning of the SARS-CoV-2 spread outside China, as well as several hospitals have changed their organization in order to increase critical care capacity and isolation areas to COVID-patients, and to generate new flows to guarantee safety and care also to non-COVID patients, also pediatric practice has changed completely.

SARS-CoV-2 is rarely involving children, and most of the time pediatric COVID-19 disease is mild. ${ }^{1}$ Moreover, parents stopped to routinely bring their children to the hospital either because of fear in contracting the infection or due to a lockdown-related drop of seasonal infectious diseases. ${ }^{2}$

As a consequence, the workload of pediatricians has significantly reduced in these months ${ }^{3}$ and therefore their inclusion in COVID-19 units should be considered by Institutions.

Although not directly involved in adult care, pediatricians may play a significant role in managing COVID19-like patients for several reasons.

From a clinical point of view, several common clinical scenarios can be included in COVID-19 adults that overlap with common pediatric conditions for which pediatricians, especially some categories of them, are already accustomed to their diagnosis and management (Table 1). ${ }^{4.5}$

In the last years, in several pediatric settings, lung ultrasound (LUS) has become the first-line imaging method in children evaluated for respiratory disease, allowing the real time diagnosis and monitoring of lung involvement. ${ }^{6-13}$

In recent years, several studies have shown that LUS is a useful and accurate tool for detecting pneumonia in children and it may be better than chest radiography in the diagnosis of community-acquired pneumonia. ${ }^{6-9,13}$ Studies are showing that LUS is able to predict more severe pneumonia, monitor antibiotic response and, recently, different LUS patterns have been able to differentiate viral from bacterial pneumonia by defining their etiology. ${ }^{6,9,13}$

Many studies have described and validated LUS scores (based mainly on vertical artifacts and subpleural consolidations) in neonatal respiratory disorders ${ }^{10}$ and bronchiolitis. ${ }^{11,12}$

For the development of lung ultrasound studies on the pediatric population but also for the development of the use of LUS in pediatric clinical practice, over the years it has been fundamental not only the references to studies performed on adults ${ }^{14,15}$ and therefore the translation in the clinical prediatric practice of knowledge 
acquired from these studies; but a true collaboration between pediatric specialists and specialists of the adult population was also fundamental, especially for the interpretation of some ultrasound findings such as in the case of vertical artifacts and ultrasound interstitial syndrome. ${ }^{7,14,15}$

Since the outbreak of the pandemic, this sharing/collaboration of experiences and studies has assumed an ever greater force that takes shape every day in daily clinical practice in the fight against COVID-19.

In particular, it is not a coincidence that a pediatrician was the one who suggested to the medical community to use ultrasound more frequently in suspected COVID-19 patients and also the one who described the first case also the one who described the first case of COVID-19 (an adult patient) diagnosed and managed with thoracic ultrasound. ${ }^{16-18}$

Since then, LUS has played a key role in the management of patients with COVID-19 pneumonia.

Importantly, the ultrasound patterns of viral pneumonia and bronchiolitis in children are similar to those seen in COVID-19 pneumonia, such as pleural line irregularities and vertical artifacts (B-lines) with patchy distribution, sub pleural consolidations and white lung areas, making easier for pediatrician to detect pathological LUS patterns in adults with COVID-19 (Figure 1).

Furthermore in general, the basic LUS semeiotics of COVID-19 pneumonia does not differs in adults and children. ${ }^{19-21}$

In order to allow comparing the severity of COVID-19 pneumonia of different patients, limiting the subjectivity and the operator-dependence of the exam, the standardization of the ultrasound semiotics of COVID-19 pneumonia and the LUS score of severity of COVID-19 pneumonia have been proposed, and in the drafting of which a pediatrician (D.B.) actively participated. ${ }^{19-20}$

Although they could face several limits dealing with adult patients, the habit to manage complex patients with multi-systemic diseases and poli-pharmacological therapies gives to pediatricians an important source of strength to contribute to the management of adult COVID-19 patients.

Probably all these strengths could also reduce the anxiety, stress and feeling of uncertainty that doctors who find themselves working in a different field from which they were trained can feel.

On the other hand, the major barrier could be represented by medico-legal concerns, as common comorbidities and complications of adult patients are far from the pediatric daily practice. A safe and effective strategy for COVID wards could be once again a multidisciplinary team. A close collaboration between pediatricians and internists (even mixing more and less experienced doctors) could be precious to re-introduce the former to adult patients and to ensure mutual clinical support.

Given their skills in pediatric infectious diseases and lung ultrasound, pediatricians D.B. and L.P. have been included in the management of adults with COVID-19 in their Institutions as well as many other colleagues in Italy.

In Bologna two internists, two pediatricians (including L.P.), one endocrinologist and a group of mixed pediatric and internist residents managed a COVID ward, for 5 weeks from March 16, 2020 to April 17, 2020. It was a 30-bed ward, with 2 negative pressure room for patients on CPAP. In those 5 weeks, a total of 145 patients were managed on oxygen therapy and CPAP. As well, other three pediatricians have been integrated in other three COVID equips. Many specialists were involved in COVID wards, but pediatricians were the only who supported actively the internists in the management of patients. The success of this collaboration derived from a common way of thinking in dealing with patients and multisystemic diseases. Even if pediatrician are not confident with adults' common pathologies, the habits to look at the patient as a whole helped to early recognize worsening signs and to facilitate the discussion about patients.

These equal views helped to reduce stress in a highly stressful situation, which could have been worse in a context where internists would have had to carry the whole work on their shoulders, while other specialists would have felt frustrated to manage situations that were risky and not congenial to them. 
In Rome, a pediatrician (D.B.) became responsible for LUS evaluation of pregnant women with respiratory conditions, in order to reduce the routine use of chest X-rays and computed tomography scans in this specific group, reserving these tools to selected cases. ${ }^{22,23}$

In particular, from March 2020 to date, 62 pregnant women positive for COVID19, have been evaluated by chest ultrasound. No positive pregnant woman has undergone a chest CT scan so far and LUS has become the first level tool for all to diagnose pneumonia.

The past, current and projected scale of distress among healthcare professionals, while understandable, has been and is of grave concern. ${ }^{9}$ The short- and long-term negative effects of this disease have the potential to have both physical and psychological consequences impacting significantly on the quality of life of both of the health worker and their family. The existing risks to the well-being of healthcare professionals are compounded under the current highly pressurized conditions. ${ }^{24}$

Concomitantly, the whole world is facing a severe shortage of Personal Protective Equipment ${ }^{3}$, which contributes to the high number of infections, disease and deaths among healthcare workers worldwide. Altogether, these factors determine an increasing shortage of doctors worldwide, including the richest countries.

Having adequate numbers of health workers and obtaining the collaboration among different specialists for whom less uncomfortable conditions possible are desired, will be critical to winning the battle against COVID-19.

Growing evidence is highlighting that COVID-19 is a systemic condition that requires doctors with multiple expertise $^{25}$ : general support measures, experience in managing antivirals, antibiotics and biological agents, respiratory support, imaging interpretation and experience in point-of -care ultrasound.

Considering the issue of staff shortage that is facing every country in the world, the complexity of COVID19 , the rare and mild involvement of SARS-CoV-2 in children ${ }^{1}$ and the reduced access to pediatric health facilities $^{2}$, the pediatricians may represent an important source of ready and skilled specialists that can quickly translate the pediatric practice in the COVID-19 care.

\section{Acknowledgements}

We are extremely grateful to the Italian Academy of Thoracic Ultrasound (Accademia Italiana Di Ecografia Toracica - ADET). All clinicians and researchers of the Academy (and dr. Gino Soldati in particular) are teaching since decades Italian doctors on the use of Lung Ultrasound, and in particular thought and inspired the youngest generation of doctors from different specialties across the country. Thanks to their teachings, we have been able to help children and adults with COVID-19 and other respiratory infections, playing our active role in this pandemic. We hope that this commentary will inspire other young colleagues and show the importance of this clinical tool.

\section{Reference}

1. Viner RM, Mytton OT, Bonell C, et al. Susceptibility to SARS-CoV-2 Infection Among Children and Adolescents Compared With Adults: A Systematic Review and Meta-analysis. JAMA Pediatr.2021;175(2):143-156.

2. Roland D, Harwood R, Bishop N, Hargreaves D, Patel S, Sinha I. Children's emergency presentations during the COVID-19 pandemic. Lancet Child Adolesc Health. 2020;4(8):e32-e33.

3. Bressan S, Buonsenso D, Farrugia R, et al. Preparedness and Response to Pediatric COVID-19 in European Emergency Departments: A Survey of the REPEM and PERUKI Networks. Ann Emerg Med.2020;76(6):788-800.

4. Mehta P, McAuley DF, Brown M, et al. HLH Across Speciality Collaboration, UK. COVID-19: consider cytokine storm syndromes and immunosuppression. Lancet. 2020;395(10229):1033-1034

5. Harwood R, Allin B, Jones CE, et al. PIMS-TS National Consensus Management Study Group. A national consensus management pathway for paediatric inflammatory multisystem syndrome temporally 
associated with COVID-19 (PIMS-TS): results of a national Delphi process.Lancet Child Adolesc Health. 2020:S2352-4642(20)30304-7.

6. Musolino AM, Tomà P, Supino MC et al. Lung ultrasound features of children with complicated and non-complicated community acquired pneumonia: A prospective study. Pediatr Pulmonol.2020;54:14791486.

7. Buonsenso D, Soldati G, Curatola A, et al. Lung Ultrasound Pattern in Healthy Infants During the First 6 Months of Life. J Ultrasound Med. 2020;39(12):2379-2388.

8. Buonsenso, D., De Rose, C., Morello, R., Lazzareschi, I., \& Valentini, P. Aspiration pneumonia in children with neurological disorders: a new indication for lung ultrasound? A case series. Journal of ultrasound. 2020;10.1007/s40477-020-00520-4.

9. Berce V, Tomazin M, Gorenjak M, Berce T, Lovrenčič B. The Usefulness of Lung Ultrasound for the Aetiological Diagnosis of Community-Acquired Pneumonia in Children. Sci Rep.2019;9(1):17957.

10. Liu J, Lovrenski J, Ye Hlaing A, Kurepa D. Neonatal lung diseases: lung ultrasound or chest X-ray. $J$ Matern Fetal Neonatal Med.2019;20:1-6.

11. Supino MC, Buonsenso D, Scateni S, et al. Point-of-care lung ultrasound in infants with bronchiolitis in the pediatric emergency department: a prospective study. Eur J Pediatr. 2019; 178:623-632.

12. Buonsenso D, Supino MC, Giglioni E, et al. Point of care diaphragm ultrasound in infants with bronchiolitis: A prospective study.Pediatr Pulmonol. 2018;53(6):778-786

13. Buonsenso D, Brancato F, Valentini P, Curatola A, Supino M, Musolino AM. The Use of Lung Ultrasound to Monitor the Antibiotic Response of Community-Acquired Pneumonia in Children: A Preliminary Hypothesis. J Ultrasound Med. 2020;39(4):817-826.

14. Soldati G, Demi M, Demi L. Ultrasound patterns of pulmonary edema.Ann Transl Med. 2019;7(Suppl 1):S16.

15. Soldati G, Demi M, Inchingolo R, Smargiassi A, Demi L. On the physical basis of pulmonary sonographic interstitial syndrome. J Ultrasound Med. 2016; 35:2075-2086.

16. Buonsenso D, Piano A, Raffaelli F, Bonadia N, de Gaetano Donati K, Franceschi F. Point-of-Care Lung Ultrasound findings in novel coronavirus disease-19 pnemoniae: a case report and potential applications during COVID-19 outbreak. Eur Rev Med Pharmacol Sci. 2020;24(5):2776-2780.

17. Buonsenso D, Pata D, Chiaretti A. COVID-19 outbreak: less stethoscope, more ultrasound. Lancet Respir Med. 2020;S2213-2600(20)30120-X

18. De Rose C, Inchingolo R, Smargiassi A, Zampino G, Valentini P, Buonsenso D. How to Perform Pediatric Lung Ultrasound Examinations in the Time of COVID-19. J Ultrasound Med 2020;39(10):20812082.

19. Soldati G, Smargiassi A, Inchingolo R, et al. Is There a Role for Lung Ultrasound During the COVID-19 Pandemic?. J Ultrasound Med2020;39(7):1459-1462.

20. Soldati G, Smargiassi A, Inchingolo R, et al. Proposal for International Standardization of the Use of Lung Ultrasound for Patients With COVID-19: A Simple, Quantitative, Reproducible Method.J Ultrasound Med 2020;39(7):1413-1419.

21. Musolino AM, Supino MC, Buonsenso D, et al. Lung ultrasound in the diagnosis and monitoring of 30 children with coronavirus disease 2019. Pediatr Pulmonol. 2021;10.1002/ppul.25255.

22. Buonsenso D, Raffaelli F, Tamburrini E, et al. Clinical role of lung ultrasound for diagnosis and monitoring of COVID-19 pneumonia in pregnant women. Ultrasound Obstet Gynecol. 2020;56(1):106109.

23. Moro F, Buonsenso D, van der Merwe J, et al. A Prospective International Lung Ultrasound Analysis Study in Tertiary Maternity Wards During the Severe Acute Respiratory Syndrome Coronavirus 2 Pandemic. J Ultrasound Med. 2020;10.1002/jum.15569.

24. Gail Kinman, Kevin Teoh, Anne Harriss. Supporting the well-being of healthcare workers during and after COVID-19. Occupational Medicine. 2020;70(5): 294-296

25. Guan W-J, Ni Z-Y, Hu Y, et al. Clinical characteristics of coronavirus disease 2019 in China. N Engl J Med. 2020;382:1708-1720

\section{Figure legend}


Figure 1 Lung ultrasound findings in children with viral lower respiratory tract infection $(\mathrm{a}, \mathrm{c})$ and in a woman with COVID-19 pneumonia (a, d), performed by the same pediatrician.

1a Lung ultrasound images of a 4 year old boy with viral pneumonia - due to Coronavirus (non-COVID-19), Bocavirus and Metapneumovirus coinfection- requiring respiratory assistance with High-flow nasal oxygen at the pediatric department; ; lung ultrasound shows areas of white lung with multiple, coalescent vertical artifacts (B-lines, black arrowhead). A similar lung ultrasound pattern (multiple vertical artifacts, B-lines, black arrowhead) was found in a 42 years old woman with COVID-19 pneumonia and moderate respiratory distress (1b ). 1c Lung ultrasound images of a 2 year old boy with H1N1 Influenza pneumonia requiring respiratory assistance with High-flow nasal oxygen at the pediatric department, showing subpleural consolidations (hypoechoic areas, black arrow) and below areas of white are evident. A similar lung ultrasound pattern (subpleural consolidations - black arrow - with areas of white lung) was found in a 31 years old pregnant woman with COVID-19 pneumonia and acute respiratory failure requiring admission in the intensive care unit (1d ).
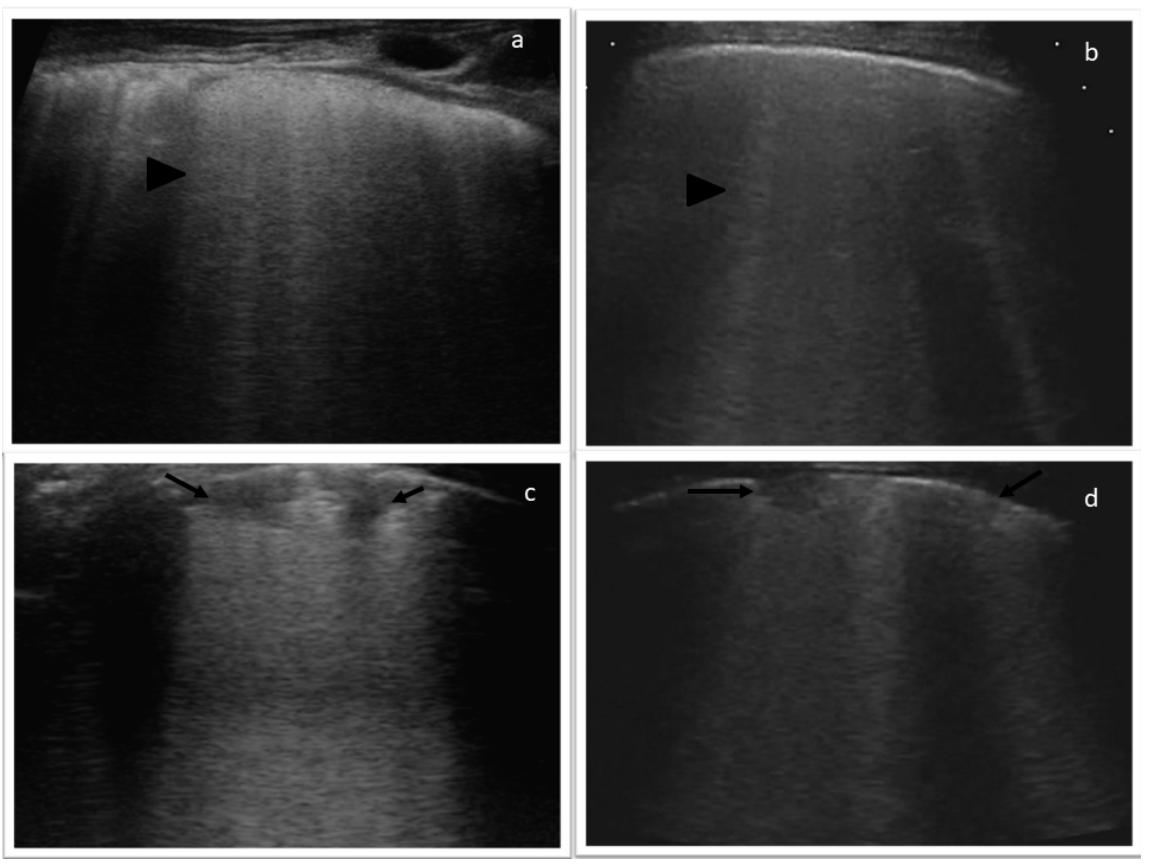

\section{Hosted file}

Table I.pdf available at https://authorea.com/users/398621/articles/514498-covid-19-andlung-ultrasound-reasons-why-pediatricians-can-support-adult-covid-19-units-duringcritical-epidemiologic-periods 